\title{
Degradación de los módulos elásticos transversal y de cortadura en materiales compuestos con refuerzo de fibra continua de carbono sometidos a fatiga
}

\author{
D. REVUELTA' ${ }^{1}$, A. RECUERO ${ }^{1}$ \\ ${ }^{1}$ Instituto de Ciencias de la Construcción Eduardo Torroja (C.S.I.C.). c/ Serrano Galvache s/n, 28033 - Madrid.
}

\begin{abstract}
La rotura a fatiga en materiales compuestos con refuerzo de fibra continua es debida en primera instancia a la aparición de grietas en la matriz que se propagan en la dirección paralela a la fibra. Es de esperar que la orientación definida de estas grietas produzca una degradación anisótropa de las propiedades elásticas de la lámina. Este trabajo caracteriza la degradación que experimenta el módulo elástico en la dirección perpendicular a la fibra de un material compuesto grafito-epoxi sometido a cargas de fatiga, hallándose que el módulo elástico sufre cambios drásticos a partir de un determinado umbral de daño, y decayendo a partir de ese punto según una ley cuadrática.
\end{abstract}

Palabras clave: fibra de carbono, daño, fatiga

\section{Degradation of the transverse and shear elastic modulii of continuous carbon fibre composite materials under fatigue}

Fatigue failure in continuous fiber composite materials is caused in first place by matrix cracks that propagate along the fiber direction. It is expected that the defined crack orientation produced an anisotropic degradation of the laminate elastic properties. This work characterizes the degradation suffered by the transverse elastic modulus of a graphite-epoxy laminate subjected to fatigue loads, concluding that the elastic modulus changes drastically from a determined damage threshold, decaying according to a quadratic law.

Keywords: carbon fibre, damage, fatigue

\section{INTRODUCCIÓN}

Es un hecho observado que las estructuras de materiales compuestos laminados de fibra continua sufren daño micro-estructural inducido por las cargas a las que están sometidas, mucho antes de que se produzca el fallo catastrófico de la estructura [1]. Como este daño no se manifiesta en cambios apreciables de las propiedades elásticas del material durante un largo periodo de tiempo, los diseñadores de estructuras de material compuesto asumen como correcto muchas veces un análisis elástico lineal complementado con un factor de seguridad adecuado para alejar el punto de rotura. Sin embargo, esta suposición es errónea. Aunque la presencia de micro-grietas estables no implica la inmediata rotura del material, la progresión de este daño determina la vida total de la estructura.

\section{DESARROLLO DEL DAÑO DURANTE LA VIDA A FATIGA}

Un laminado típico de matriz frágil con refuerzo de fibra continua generalmente sufre tres modos de daño distinguibles y significativos: rotura de la matriz; delaminación; y rotura de fibras.

Normalmente, antes de la delaminación aparece un gran número de grietas en la matriz (Fig. 1). La culminación de esta fase del daño se conoce como el estado de daño característico [3], ya que parece llegarse a este estado independientemente de la historia de cargas. La delaminación aparece después como resultado de las concentraciones de tensiones en las intersecciones entre las grietas y las láminas adyacentes. Finalmente, la aparición de la delaminación a gran escala en todo el laminado produce tensiones suficientes como para romper las fibras, lo que lleva a una rotura inestable. Hay una gran cantidad de literatura que describe las observaciones experimentales de estos aspectos [4-7].

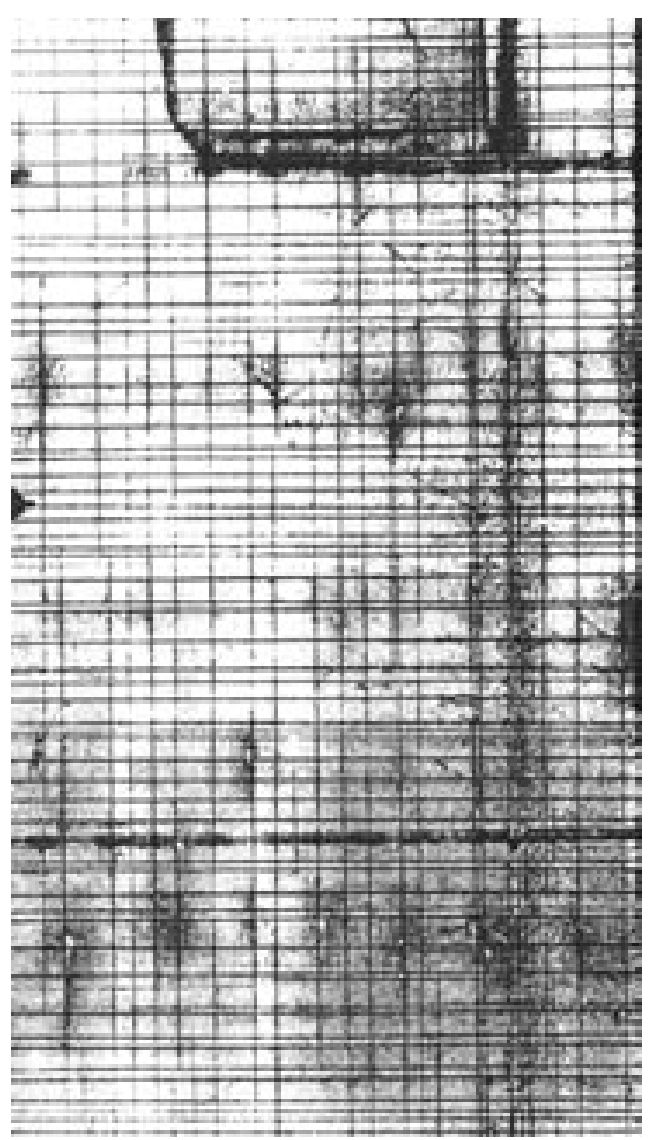

Figura 1. Radiografía de rayos $\mathrm{X}$ que muestra el daño en un laminado [0/90] de grafito-epoxi (Jamison y otros [2]) 
Ya que la primera fase del daño es la de grietas en la matriz, parece lógico centrarse primero en su caracterización. Como las grietas se producen a una escala muy pequeña comparada con la geometría de un elemento estructural, es adecuado emplear consideraciones de la mecánica del daño continuo para tratar de caracterizarlas. Esta aproximación, propuesta en primer lugar por Kachanov [8], y desarrollada posteriormente por Rabotnov [9], trata de describir el fenómeno del daño mediante el concepto de discontinuidad, en lo que constituye la primera variable que puede ser interpretada como el daño en el medio continuo. Este parámetro se define como la fracción de huecos existentes en la sección del sólido.

$$
D=\frac{A_{v}}{A}
$$

donde $A_{v}$ es el área ocupada por los huecos en el plano de área $A$ normal a la dirección de aplicación de la tensión máxima. De esta definición se obtiene un nuevo concepto de tensión, la tensión efectiva, definida como:

$$
\bar{\sigma}=\frac{\sigma}{1-D}
$$

Lemaitre y Chaboche [10], establecen la hipótesis de que el efecto de las microgrietas se puede homogeneizar en la escala local para pasar luego a la escala del elemento estructural. Sin entrar en más desarrollo teórico, consideraron el efecto que los huecos y las microgrietas tenían sobre las constantes elásticas del material, mediante la definición del Principio de Deformación Equivalente:

"Cualquier ecuación elástica constitutiva en un material dañado puede derivarse de la misma manera en el material virgen mediante la sustitución de la tensión ingenieril por la tensión efectiva"

Si por simplicidad consideramos el caso unidireccional, e identificamos al módulo de Young del material dañado mediante $\overline{\mathrm{E}}$, el módulo inicial o sin dañar por $\mathrm{E}$, $\mathrm{y}$ a la tensión efectiva mediante $\bar{\sigma}$, tenemos que

$$
\begin{aligned}
& \sigma=\overline{\mathrm{E}} \varepsilon \\
& \bar{\sigma}=\mathrm{E} \varepsilon
\end{aligned}
$$

Por tanto, la relación entre la tensión efectiva en la configuración ficticia y la tensión real en la configuración dañada viene dada por

$$
\bar{\sigma}=\left(\frac{E}{\bar{E}}\right) \sigma
$$

Si empleamos de nuevo la definición de tensión efectiva de Kachanov-Rabotnov, se obtiene

$$
D=1-\frac{\bar{E}}{E}
$$

Por tanto, el daño se puede medir a través de los cambios en el módulo del material.

\section{PARTE EXPERIMENTAL}

Los ensayos se realizaron sobre probetas tubulares de carbonoepoxi T300/862 fabricadas mediante enrollamiento continuo, laminadas a $90^{\circ}$ respecto al eje del tubo. Las probetas se sometieron a ensayos de fatiga de tracción y cortadura, aplicando una tracción en la dirección del eje del tubo (tensión transversal a la fibra), o un momento en torno al eje del tubo (tensión de cortadura). La novedad de este ensayo es que geometría tubular permite conseguir tensiones de tracción y cortadura puras, ya que desaparecen los efectos de borde libre. Con objeto de reducir la concentración de tensiones en las mordazas, se enrollaron varias capas de vidrio epoxi en la sección de amarre, mecanizándolas posteriormente para formar una lengüeta de amarre de $5^{\circ}$ de inclinación respecto al eje vertical. Los ensayos se llevaron a cabo en una máquina servo-hidráulica universal, con control de carga, y para frecuencias de $2 \mathrm{~Hz}$. La carga y la deformación se registraron a intervalos regulares por medio de un extensómetro multiaxial especialmente diseñado para medir deformaciones axiales y de cortadura. Las cargas de rotura se eligieron basándose en resultados de fatiga previos, tratando de no sobrepasar una vida a fatiga de $10^{6}$ ciclos. Los datos del ensayo se muestran en la Tabla 1

TABLA 1. LÍMITES DE ROTURA Y UMBRAL DE DAÑO

\begin{tabular}{|l|c|c|c|}
\hline \multirow{2}{*}{ Ensayo } & Carga aplicada & $\begin{array}{c}\text { Límite de rotura } \\
\text { de daño, } \boldsymbol{D}_{f}\end{array}$ & $\begin{array}{c}\text { Umbral } \\
\text { de daño, } \boldsymbol{N}_{\boldsymbol{D}}\end{array}$ \\
\hline \multirow{2}{*}{$\begin{array}{l}\text { Tracción } \\
\text { pura }\end{array}$} & $\sigma_{\max }=0,75 \sigma_{\text {UTS }}$ & 0,24 & $\approx 7 \times 10^{1}$ ciclos \\
\cline { 2 - 4 } & $\sigma_{\max }=0,60 \sigma_{\text {UTS }}$ & 0,18 & $\approx 1 \times 10^{4}$ ciclos \\
\hline \multirow{2}{*}{$\begin{array}{l}\text { Cortadura } \\
\text { pura }\end{array}$} & $\sigma_{\max }=0,75 \sigma_{\text {UTS }}$ & 0,18 & $\approx 3 \times 10^{1}$ ciclos \\
\cline { 2 - 4 } & $\sigma_{\max }=0,45 \sigma_{\text {UTS }}$ & 0,21 & $\approx 1 \times 10^{5}$ ciclos \\
\hline
\end{tabular}

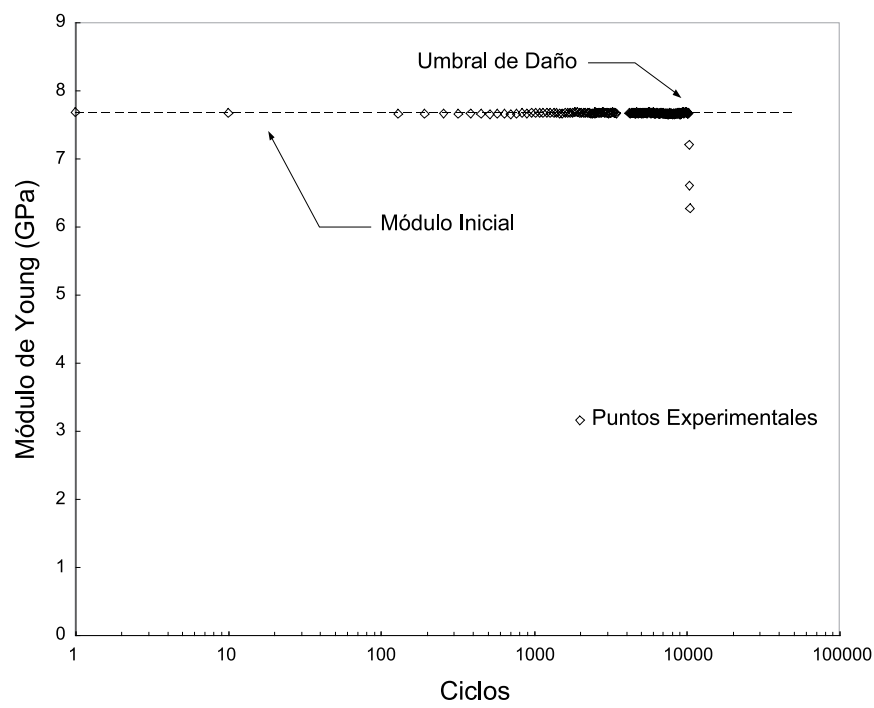

Figura 2. Evolución de la rigidez transversal en un laminado unidireccional T300/862 orientado a $90^{\circ}$ respecto al eje de carga durante la vida de fatiga en un ensayo tracción-tracción $\left(\sigma_{\max }=0,75 \sigma_{\mathrm{UTS}} ; \mathrm{R}=0 ; \omega=2 \mathrm{~Hz}\right)$

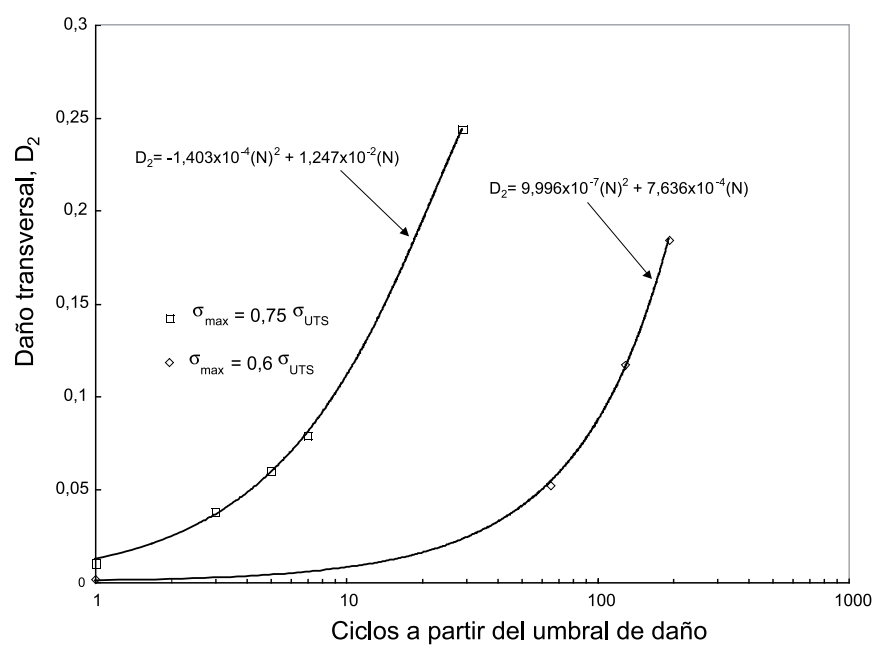

Figura 3. Evolución del daño transversal a fatiga en un laminado unidireccional grafito-epoxi $\mathrm{T} 300 / 862$ orientado a $90^{\circ}$ respecto al eje de carga a partir del umbral de daño y hasta la rotura, para diferentes niveles de tensión $\sigma_{\text {max }}(\mathrm{R}=0 ; \omega=2 \mathrm{~Hz})$ 


\section{RESULTADOS}

La Figura 2 muestra los valores del módulo de Young del laminado unidireccional grafito-epoxi $\mathrm{T} 300 / 862$ orientado a $90^{\circ}$ respecto al eje de carga, medido experimentalmente en varios ciclos. El eje de ordenadas representa el valor del módulo elástico en la dirección de aplicación de la carga, que coincide con el módulo transversal del material compuesto, $\mathrm{E}_{2}$. De la Figura 2 se desprende que la pérdida de rigidez no es inmediata, sino que existe un umbral de daño, $N_{\mathrm{D}^{\prime}}$ a partir del cual se aprecia la degradación en el módulo elástico transversal. Otro punto interesante es el punto final de rotura, que tiene lugar para un valor límite del daño en el material, $D_{f}$. La Figura 3 muestra la evolución del daño transversal en un material compuesto T300/862 unidireccional durante la última parte de la vida a fatiga de la lámina, es decir, a partir del umbral de daño del material. El daño se determina mediante la variación en el módulo elástico transversal.

La forma de las curvas nos permite deducir que el daño producido por cargas cíclicas aplicadas perpendicularmente a la dirección principal de la fibra en un laminado unidireccional evoluciona a partir de un número de ciclos según una ley cuadrática según la Ecuación 7 , en donde $A$ y $B$ son constantes que dependen de la tensión máxima aplicada, $\sigma_{\max }$.

$$
\mathrm{D}_{2}=\mathrm{A} \mathrm{N}^{2}+\mathrm{BN}
$$

La Figura 4 muestra la evolución del daño por cortadura en el laminado unidireccional grafito-epoxi T300/862 sometido a cargas de fatiga por cortadura. De nuevo el daño se determina mediante la variación del módulo elástico. Como en el caso en que el material estaba sometido a cargas de tracción transversales, el daño por cortadura parece seguir una ley cuadrática de la forma

$$
\mathrm{D}_{6}=\mathrm{CN}^{2}+\mathrm{DN}
$$

a partir del momento en que se sobrepasa el umbral de daño. Las constantes $C$ y $D$ dependen de la tensión máxima aplicada

\section{CONCLUSIONES}

- El daño por fatiga produce una degradación de las propiedades elásticas del material, según lo predicho por la mecánica del daño, que es posible registrar mediante la apropiada extensometría.

- Se observa que existe un umbral de daño, $N_{\mathrm{D}^{\prime}}$ a partir del cual es apreciable la degradación de las propiedades elásticas, lo que indica que los mecanismos de daño se ven precedidos por una fase previa de iniciación.

- Por observación experimental, se establece una ley cuadrática que describe el crecimiento del daño producido por la fatiga en un material compuesto de fibra continua a partir del mencionado umbral de daño, $N_{\mathrm{D}^{\prime}}$ bajo cargas tanto transversales a la fibra como de cortadura.

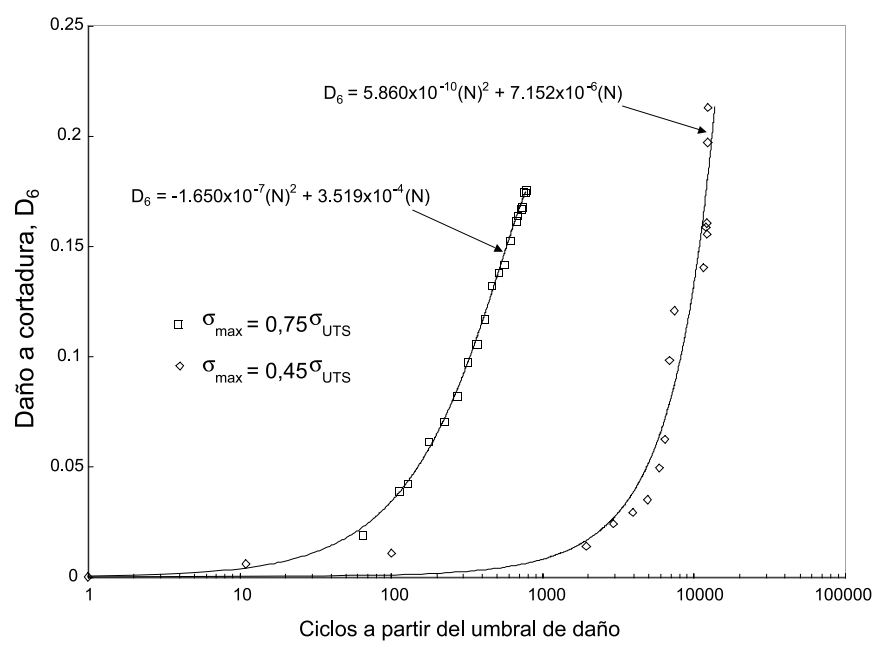

Figura 4. Evolución del daño por cortadura en el laminado unidireccional grafito-epoxi T300/ 862 sometido a cargas de fatiga por cortadura.

- La rotura final se produce para valores similares de daño, $D_{\rho}$ lo que parece indicar que el nivel de degradación es parejo en todos los especimenes, independientemente del tipo de carga aplicada y del nivel de tensión.

\section{BIBLIOGRAFÍA}

1. Allen, D.H., “Damage evolution in laminates”, Composite Material Series, Vol. 9: Damage Mechanics of Composite Materials, Ed. R. Talreja, Elsevier, 1994, pp. 79-117

2. Jamison, R.D., Schulte, K., Reifsnider, K.L., Stinchcomb, W.W., “Effects od Defects in Composite Materials", ASTM STP 836, American Society for Testing and Materials, 1984, pp. 21-55

3. Reifsnider, K.L., "Some fundamental aspects of the fatigue and fracture response if composite materials", Proc. $4^{\text {th }}$ Annu. Meeting Society Engineering Science, 1977, pp. 14-16

4. Stinchcomb, W.W., Reifsnider, K.L., "Fatigue damage mechanisms in composite materials: a review", Fatigue Mechanism, Proc. ASTM-NBS-NBF Symp., ASTM STP 679, 1979, pp. 762-787

5. Stinchcomb, W.W., Reifsnider, K.L., Yeung, P., Masters, J.E., “Effect of ply constraint on fatigue damage development in composite materials laminates", Fatigue of Fibrous Continuous Materials, ASTM STP 723, 1981, pp. 187197

6. Reifsnider, K.L., Jamison, R.D., “Fracture of fatigue-loaded composites”, Int. J. Fatigue, 1982, pp. 187-197

7. Talreja, R., Fatigue of Composite Materials, Echnomic Publishing, 1987

8. Kachanov, L.M., “On the creep fracture time”, Izv. Acad. Nauk SSSR, Otd. Techn. Nauk, No. 8, 1958, 26-31.

9. Rabotnov, Y.N., Creep Problems in Structural Members, North Holland, Amsterdam, 1969.

10. Lemaitre, J., Chaboche, J., “Áspect phenomenologique de la rupture par endommagement", J. Mécanique Appliquee, 2, No 3, 1978, 317-365.

Recibido: 1.2.03

Aceptado: 30.11 .03 